\title{
Reachability of a class of discrete-time positive switched systems
}

\author{
Ettore Fornasini and Maria Elena Valcher
}

\begin{abstract}
In this paper, monomial reachability and reachability of a special class of discrete-time positive switched systems are investigated. Necessary and sufficient conditions for these properties to hold, together with some related examples, are provided.
\end{abstract}

\section{INTRODUCTION}

Switched linear systems have attracted the interest of several scientists, in the last fifteen years. Initially treated as special cases of the broader class of hybrid systems, they have later gained complete autonomy and have been the object of an in-depth analysis. While the first contributions were almost exclusively concentrated on the stability and stabilizability properties [12], [18], nowadays several other issues have been investigated and, in particular, structural properties, like reachability, controllability and observability have been explored [9], [15], [17], [19].

Despite of the numerous research efforts, these issues still offer a quite interesting set of open problems. Indeed, structural properties have found a rather complete characterization for the class of continuous-time switched systems and for the class of reversible discrete-time switched systems (by this meaning that the system matrices of all the subsystems among which the system commutes are nonsingular). The non-reversible discrete-time case, however, still deserves investigation, since necessary and sufficient conditions for reachability (and observability) have been provided only under certain structural constraints (see, e.g., [7]). However, it must be pointed out that some interesting properties of the controllable sets for (both reversible and non-reversible) discrete-time switched systems have been investigated in the pioneering works of Conner and Stanford [10], [11], [14].

Positive linear systems, on the other hand, are state-space models in which the state variables are always positive, or at least nonnegative, in value. These systems have received considerable attention in the literature, as they naturally arise in various fields such as bioengineering (compartmental models), economic modelling, behavioral science, and stochastic processes (Markov chains), where the state variables represent quantities that may not have meaning unless nonnegative. In the last two decades, several system issues have been addressed for positive systems, by taking advantage of the powerful tools coming out of positive matrix theory and, even more, of graph theory. In particular, the analysis of controllability and reachability properties of positive discrete-time systems has been the object of a noteworthy interest [3], [6], [16].

Ettore Fornasini and Maria Elena Valcher are with the Dipartimento di Ingegneria dell'Informazione, Università di Padova, via Gradenigo 6/B, 35131 Padova, Italy, \{fornasini, meme\}@dei.unipd.it.
Switched positive systems deserve investigation both for theoretical reasons and for practical applications. Indeed, switching among different system models naturally arises as a way to mathematically formalize the fact that the system laws change under different operating conditions. Indeed, different discrete-time positive systems, which arise when discretizing linear differential equations describing processes whose state variables are temperatures, pressures, population levels, etc., or when providing a discrete-time model for the time evolution of productions levels or stocked amounts of some good, may undergo different working conditions and, consequently, switch among different mathematical models.

The aim of this paper is to define monomial reachability and reachability properties for a special class of discretetime positive switched systems, for which the system matrix is the same, while the input-to-state matrix changes, and to provide some necessary and sufficient conditions for these properties to hold.

Before proceeding, we introduce some notation. Given any positive integer $k \in \mathbb{N}$, we denote by $\langle k\rangle$ the set of positive integers $\{1,2, \ldots, k\}$. The symbol $\mathbb{R}_{+}$denotes the semiring of nonnegative real numbers. A matrix $M \in \mathbb{R}_{+}^{n \times m}$ is said to be a nonnegative matrix; if $M$ is nonnegative and nonzero it is called positive, while if all its entries are greater than zero, $M$ is strictly positive. The $(i, j)$ th entry of a matrix $M$ is denoted by $[M]_{i j}$, while the $i$ th entry of a vector $v$ is $[v]_{i}$. We let $\mathbf{e}_{i}$ denote the $i$ th vector of the canonical basis in $\mathbb{R}^{n}$ (where $n$ is always clear from the context). Given a nonnegative vector $v$, the nonzero pattern of $v$ is the set of indices corresponding to its nonzero entries, namely $\overline{\mathrm{ZP}}(v):=\left\{i:[v]_{i} \neq 0\right\}$. A vector $v \in \mathbb{R}_{+}^{n}$ is an ith monomial vector if $\overline{\mathrm{ZP}}(v)=\overline{\mathrm{ZP}}\left(\mathbf{e}_{i}\right)=\{i\}$. A monomial matrix is a nonsingular square nonnegative matrix whose columns are (of course, distinct) monomial vectors. A monomial matrix whose nonzero entries are all unitary is called permutation matrix.

Given an $n$-dimensional positive system with $p$ inputs, namely a state-space model

$$
x(k+1)=A x(k)+B u(k), \quad k \in \mathbb{Z}_{+},
$$

where $x(\cdot)$ and $u(\cdot)$ denote the $n$-dimensional nonnegative state variable and the $p$-dimensional nonnegative input variable, $A \in \mathbb{R}_{+}^{n \times n}$ and $B \in \mathbb{R}_{+}^{n \times p}$, we may associate with it [3], [4], [16] a digraph (directed graph) $\mathcal{D}(A, B)$, with $n$ vertices, indexed by $1,2, \ldots, n$, and $p$ sources $s_{1}, s_{2}, \ldots, s_{p}$. There is an arc $(j, i)$ from $j$ to $i$ if and only if $[A]_{i j}>0$, 
and there is an arc $\left(s_{j}, i\right)$ from the source $s_{j}$ to the vertex $i$ if and only if $[B]_{i j}>0$.

A sequence $s_{j} \rightarrow i_{0} \rightarrow i_{1} \rightarrow \ldots \rightarrow i_{k-1}$, starting from the source $s_{j}$, and passing through the vertices $i_{0}, \ldots, i_{k-1}$, is an $s$-path from $s_{j}$ to $i_{k-1}$ (of length $k$ ) provided $\left(s_{j}, i_{0}\right),\left(i_{0}, i_{1}\right), \ldots,\left(i_{k-2}, i_{k-1}\right)$ are all arcs of $\mathcal{D}(A, B)$. An $s$-path of length $k$ from $s_{j}$ deterministically reaches some vertex $i$, if no other vertex of the digraph can be reached in $k$ steps starting from $s_{j}$. It is easily seen that there is an $s$-path of length $k$ from $s_{j}$ to $i$ if and only if the $(i, j)$ th entry of $A^{k-1} B$ is positive. In general, leaving from $s_{j}$, after $k$ steps one can reach several distinct vertices: this corresponds to saying that the $j$ th column of $A^{k-1} B$ can have more than one nonzero entry. So, a vertex $i$ can be deterministically reached from the source $s_{j}$ by means of a path of length $k$ if and only if the $j$ th column of $A^{k-1} B$ is an $i$ th monomial vector.

Basic definitions and results about cones may be found, for instance, in [1], [2]. We recall here only those facts that will be used within this paper.

A set $\mathcal{K} \subset \mathbb{R}^{n}$ is said to be a cone if $\alpha \mathcal{K} \subseteq \mathcal{K}$ for all $\alpha \geq 0$; a cone is convex if it contains, with any two points, the line segment between them. A cone $\mathcal{K}$ is said to be polyhedral if it can be expressed as the set of nonnegative linear combinations of a finite set of generating vectors. This amounts to saying that a positive integer $k$ and an $n \times k$ matrix $C$ can be found, such that $\mathcal{K}$ coincides with the set of nonnegative combinations of the columns of $C$. In this case, we adopt the notation $\mathcal{K}:=\operatorname{Cone}(C)$.

\section{DisCRETE-TIME SINGLE-INPUT POSITIVE SWITCHED SYSTEMS AND REACHABILITY PROPERTIES}

A discrete-time single-input positive switched system is described, at each time instant $t \in \mathbb{Z}_{+}$, by a first-order difference equation of the following type:

$$
x(t+1)=A_{\sigma(t)} x(t)+b_{\sigma(t)} u(t),
$$

where $x(\cdot)$ and $u(\cdot)$ denote the $n$-dimensional nonnegative state variable and the nonnegative scalar input, while $\sigma$ is a switching sequence, defined on $\mathbb{Z}_{+}$and taking values in a finite set $\mathcal{P}:=\langle p\rangle$. For each $i \in \mathcal{P}$, the pair $\left(A_{i}, b_{i}\right)$ represents a discrete-time single-input positive system (1), which means that $A_{i} \in \mathbb{R}_{+}^{n \times n}$ and $b_{i} \in \mathbb{R}_{+}^{n}$.

The definitions of monomial reachability and of reachability for discrete-time positive switched systems may be given by suitably adjusting the definitions of reachability given in [9], [19], in order to introduce the nonnegativity constraint on the state and input variables.

Definition 1: [13] A state $x_{f} \in \mathbb{R}_{+}^{n}$ is said to be reachable at time $k \in \mathbb{N}$ if there exist a switching sequence $\sigma: \mathbb{Z}_{+} \rightarrow$ $\mathcal{P}$ and an input sequence $u: \mathbb{Z}_{+} \rightarrow \mathbb{R}_{+}$that lead the state trajectory from $x(0)=0$ to $x(k)=x_{f}$.

System (2) is said to be monomially reachable if every monomial vector $x_{f} \in \mathbb{R}_{+}^{n}$ (equivalently, every vector $\mathbf{e}_{i}$ of the canonical basis in $\mathbb{R}^{n}$ ) is reachable at some time instant $k$. System (2) is said to be reachable if every state $x_{f} \in \mathbb{R}_{+}^{n}$ is reachable at some time instant $k$.
Since (monomial) reachability always refers to a finite time interval, focusing on the value of the state at the final instant $k$, only the values of the switching sequence $\sigma$ (and of the input sequence $u$ ) within $\{0,1, \ldots, k-1\}$ are relevant. So, we refer to the cardinality of the discrete time interval $\{0,1, \ldots, k-1\}$ as to the length of the switching sequence $\sigma$ and we denote it by $|\sigma|$ (in this case, $|\sigma|=k$ ).

When (monomial) reachability property is ensured, a natural goal one may want to pursue is that of determining the maximum number of steps required to reach every (monomial) nonnegative state. This leads to the following definition of (monomial) reachability index.

Definition 2: Given a (monomially) reachable switched system (2), we define its (monomial) reachability index as $\left(I_{M R}:=\max _{i \in\langle n\rangle} \min \left\{k: \mathbf{e}_{i}\right.\right.$ is reachable at time $\left.\left.k\right\}\right)$ $I_{R}:=\sup _{x \in \mathbb{R}_{+}^{n}} \min \{k: x$ is reachable at time $k\}$.

It has been shown in [13] that reachable systems can be found endowed with an infinite ${ }^{1} I_{R}$. Also, for monomially reachable systems the index $I_{M R}$ can far exceed the system dimension and even reach the theoretical bound of $2^{n}-1$. These facts represent significant differences with respect to both standard switched systems and positive systems.

\section{MONOMIAL REACHABILITY OF A CLASS OF SINGLE-INPUT POSITIVE SWITCHED SYSTEMS}

In this paper we consider the special class of discretetime single-input positive switched systems described by the following state equation

$$
x(t+1)=A x(t)+b_{\sigma(t)} u(t) .
$$

The special property of this class of systems is that its $p=$ $|\mathcal{P}|$ subsystems share the same positive state matrix $A$, and hence differ only in the input-to-state matrix, $b_{i} \in \mathbb{R}_{+}^{n}, i \in \mathcal{P}$. For these systems we will provide characterizations of both monomial reachability and of reachability, and we will show that the indices $I_{M R}$ and $I_{R}$ are always upper bounded by the system dimension $n$.

To explore monomial reachability and reachability for the class of systems (3), it is first convenient to provide the explicit expression of the state at any time instant $k \in \mathbb{N}$, starting from the initial condition $x(0)=0$, under the effect of the input sequence $u(0), u(1), \ldots, u(k-1)$, and of the switching sequence $\sigma(0), \sigma(1), \ldots, \sigma(k-1)$. It turns out that

$$
\begin{aligned}
x(k) & =A^{k-1} b_{\sigma(0)} u(0)+A^{k-2} b_{\sigma(1)} u(1)+\ldots \\
& +A b_{\sigma(k-2)} u(k-2)+b_{\sigma(k-1)} u(k-1),
\end{aligned}
$$

where $\sigma(i) \in \mathcal{P}$ for every $i \in\{0,1, \ldots, k-1\}$. If we define the reachability matrix associated with the switching sequence $\sigma$ of length $k$ as

$\mathcal{R}_{k}(\sigma)=\left[\begin{array}{llll}A^{k-1} b_{\sigma(0)} & A^{k-2} b_{\sigma(1)} & \ldots A b_{\sigma(k-2)} & b_{\sigma(k-1)}\end{array}\right]$,

\footnotetext{
${ }^{1}$ It is worthwhile to underline that even when $I_{R}$ is infinite, each single nonnegative state can be reached in a finite number of steps. However, such a number of steps may be arbitrarily high. This concept must not be confused with the weak reachability property of positive systems [16], which allows to reach certain states only asymptotically.
} 
it is easily seen that (4) can be rewritten as

$$
x(k)=\mathcal{R}_{k}(\sigma)\left[\begin{array}{c}
u(0) \\
\vdots \\
u(k-1)
\end{array}\right],
$$

and hence $x(k) \in \operatorname{Cone}\left(\mathcal{R}_{k}(\sigma)\right)$. This is a much simpler expression w.r.t. the general one available for system (2). Nonetheless, as we will see, even for this class of systems, reachability characterization turns out to be articulate. Clearly, a positive state $x_{f}$ is reachable if and only if there exists a switching sequence $\sigma$ such that $x_{f} \in$ $\operatorname{Cone}\left(\mathcal{R}_{|\sigma|}(\sigma)\right)$. Equivalently, $x_{f}$ is reachable if and only if there exist $k \in \mathbb{N}, i_{1}, i_{2}, \ldots, i_{k} \in \mathcal{P}$, such that

$$
x_{f} \in \text { Cone }\left(\left[\begin{array}{lllll}
A^{k-1} b_{i_{k}} & A^{k-2} b_{i_{k-1}} & \ldots & A b_{i_{2}} & b_{i_{1}}
\end{array}\right]\right) .
$$

As a first step, we derive the characterization of monomial reachability.

Proposition 1: Given a discrete-time single-input positive switched system described by the state equation (3), the following facts are equivalent ones:

i) the switched system (3) is monomially reachable;

ii) the $n$-dimensional non-switched positive system with $p$ inputs (1), described by the pair $(A, B)$, with

$$
B:=\left[\begin{array}{llll}
b_{1} & b_{2} & \ldots & b_{p}
\end{array}\right],
$$

is reachable, namely [5], [16] its reachability matrix

$$
\mathcal{R}_{n}(A, B):=\left[\begin{array}{llll}
A^{n-1} B & \ldots & A B & B
\end{array}\right],
$$

contains an $n \times n$ monomial submatrix.

So, if system (3) is monomially reachable, then its monomial reachability index $I_{M R}$ can never exceed $n$.

Proof: System (3) is monomially reachable if and only if for every $i \in\langle n\rangle$ there exists a switching sequence $\sigma_{i}$ of length say $k_{i}$ such that $\mathbf{e}_{i} \in \operatorname{Cone}\left(\mathcal{R}_{k_{i}}\left(\sigma_{i}\right)\right)$. But this amounts to saying that for every $i \in\langle n\rangle$ there exists $h_{i} \in \mathbb{Z}_{+}$and $j_{i} \in \mathcal{P}$ such that $A^{h_{i}} b_{j_{i}}$ is an $i$ th monomial vector. As proved in [5], if such an index $h_{i}$ exists, than it can always be chosen not greater than $n-1$. But this ensures, that all monomial vectors are reachable if and only if $\mathcal{R}_{n}\left(A,\left[\begin{array}{lll}b_{1} & \ldots & b_{p}\end{array}\right]\right)$ contains $n$ linearly independent monomial vectors and hence an $n \times n$ monomial matrix.

The final statement about $I_{M R}$ is an obvious consequence.

Before moving to the characterization of reachability, we aim now to provide some meaningful examples, illustrating how even for this simple class of positive switched systems several complex situations arise w.r.t. standard positive systems. As a first thing, monomial reachability is not equivalent to reachability. This is shown by the following elementary counterexample.

Example 1: Consider the discrete-time single-input positive switched system described by the state equation (3), with

$$
A=0_{2 \times 2}, \quad b_{1}=\left[\begin{array}{l}
1 \\
0
\end{array}\right], \quad b_{2}=\left[\begin{array}{l}
0 \\
1
\end{array}\right]
$$

(so that $\mathcal{P}=\langle 2\rangle$ ). It is easily seen that all monomial vectors are reachable, but no vector with both positive entries can be reached. So, there is monomial reachability, but not reachability.

The reason why monomial reachability does not ensure reachability is because different monomial vectors can be reached along different switching sequences and such switching sequences are not necessarily compatible. Of course, if we can find a single switching sequence along which one may reach every monomial vector, then reachability is ensured.

Proposition 2: Given a discrete-time single-input positive switched system described by the state equation (3), if there exists a switching sequence $\sigma$ of length say $k$ such that the reachability matrix $\mathcal{R}_{k}(\sigma)$ includes an $n \times n$ monomial submatrix, then the system is reachable (with $I_{R} \leq k$ ).

Proof: Obvious, as in this case it would be $\operatorname{Cone}\left(\mathcal{R}_{k}(\sigma)\right)=\mathbb{R}_{+}^{n}$.

It is worthwhile noticing that the existence of a switching sequence $\sigma$ of length say $k$ such that the reachability matrix $\mathcal{R}_{k}(\sigma)$ includes an $n \times n$ monomial submatrix corresponds to the possibility of choosing $n$ distinct indices $h_{1}, h_{2}, \ldots, h_{n} \in \mathbb{Z}_{+}$such that $\overline{\mathrm{ZP}}\left(A^{h_{i}} b_{j_{i}}\right)=\{i\}$ for suitable indices $j_{i} \in \mathcal{P}$. In fact, if this is the case, by simply choosing a switching sequence $\sigma$ of length $k:=$ $\max _{i \in\langle n\rangle}\left\{h_{i}\right\}+1$, which satisfies $\sigma\left(k-1-h_{i}\right)=j_{i}$, for $i \in\langle n\rangle$, and that takes arbitrary values at all time instants $t \in\{0,1, \ldots, k-1\} \backslash\left\{h_{1}, h_{2}, \ldots, h_{n}\right\}$, we ensure that $\mathcal{R}_{k}(\sigma)$ includes an $n \times n$ monomial submatrix.

Example 2: Consider the discrete-time single-input positive switched system described by the state equation (3), with

$$
A=\left[\begin{array}{llllll}
0 & 1 & 0 & 0 & 0 & 0 \\
0 & 0 & 1 & 0 & 0 & 0 \\
0 & 0 & 0 & 0 & 0 & 0 \\
2 & 0 & 0 & 0 & 1 & 0 \\
0 & 0 & 0 & 0 & 0 & 1 \\
1 & 0 & 0 & 1 & 0 & 0
\end{array}\right], b_{1}=\left[\begin{array}{l}
0 \\
0 \\
1 \\
0 \\
0 \\
0
\end{array}\right], b_{2}=\left[\begin{array}{l}
0 \\
0 \\
0 \\
0 \\
0 \\
1
\end{array}\right] \text {. }
$$

It is easily seen that the system is monomially reachable, since

$$
b_{1}=\mathbf{e}_{3}, A b_{1}=\mathbf{e}_{2}, A^{2} b_{1}=\mathbf{e}_{1}
$$

while

$$
b_{2}=\mathbf{e}_{6}, A b_{2}=\mathbf{e}_{5}, A^{2} b_{2}=\mathbf{e}_{4} .
$$

On the other hand, it is easily seen that $\mathbf{e}_{4}, \mathbf{e}_{5}, \mathbf{e}_{6}$ can also be obtained as

$$
A^{3} b_{2}=\mathbf{e}_{6}, A^{4} b_{2}=\mathbf{e}_{5}, A^{5} b_{2}=\mathbf{e}_{4},
$$

and hence by choosing $\sigma$ of length 6 , taking value 2 at $k=$ $0,1,2$, and taking value 1 at $k=3,4,5$, we get

$$
\mathcal{R}_{6}(\sigma)=\left[\mathbf{e}_{4}, \mathbf{e}_{5}, \mathbf{e}_{6}, \mathbf{e}_{1}, \mathbf{e}_{2}, \mathbf{e}_{3}\right] .
$$

This ensures reachability. 
The condition provided in Proposition 2 above is only sufficient for the system reachability. In fact, reachability property may be ensured, but different positive vectors can be reached only by means of different switching sequences. It is worthwhile to remark, however, that for standard (i.e., nonpositive) switched systems the situation is different. Indeed, it has been proved [9] that a switched system is reachable if and only if there exists a switching sequence $\sigma$ (of length say $k$ ) such that $\operatorname{Im}\left(\mathcal{R}_{k}(\sigma)\right)=\mathbb{R}^{n}$.

Example 3: Consider the discrete-time single-input positive switched system described by the state equation (3), with

$$
A=\left[\begin{array}{ll}
2 & 2 \\
3 & 1
\end{array}\right], \quad b_{1}=\left[\begin{array}{l}
1 \\
0
\end{array}\right], \quad b_{2}=\left[\begin{array}{l}
0 \\
1
\end{array}\right] .
$$

It is easily seen that the system is monomially reachable, even more it is reachable (with finite reachability index). Indeed,

$$
\operatorname{Cone}\left(\left[\begin{array}{ll}
b_{1} & A b_{1}
\end{array}\right]\right)=\text { Cone }\left(\left[\begin{array}{ll}
1 & 2 \\
0 & 3
\end{array}\right]\right),
$$

while

$$
\text { Cone }\left(\left[\begin{array}{ll}
b_{2} & A b_{2}
\end{array}\right]\right)=\text { Cone }\left(\left[\begin{array}{ll}
0 & 2 \\
1 & 1
\end{array}\right]\right) \text {. }
$$

So, if we define $\sigma_{1}$ the switching sequence of length 2 taking only value 1 , and $\sigma_{2}$ the switching sequence of length 2 taking only value 2 , we get

$$
\operatorname{Cone}\left(\mathcal{R}_{2}\left(\sigma_{1}\right)\right) \cup \operatorname{Cone}\left(\mathcal{R}_{2}\left(\sigma_{2}\right)\right)=\mathbb{R}_{+}^{2},
$$

which ensures reachability. On the other hand, there is no way to find a switching sequence $\sigma$, of length say $k$, such that $\mathcal{R}_{k}(\sigma)$ includes a $2 \times 2$ monomial submatrix. In fact, $\mathbf{e}_{i}=b_{i}$ for $i \in\langle 2\rangle$, however for every $k \in \mathbb{N}$ and every $i \in\langle 2\rangle,\left|\overline{\mathrm{ZP}}\left(A^{k} b_{i}\right)\right|>1$.

To conclude, we present a preliminary result which will be useful for providing a characterization of reachability in the following section. This lemma represents a refinement of Lemma 2 in [16].

Lemma 1: If the positive switched system (3) is reachable, then the $n \times(n+p)$ positive matrix $\left[\begin{array}{lllll}A & b_{1} & b_{2} & \ldots & b_{p}\end{array}\right]$ includes an $n \times n$ monomial matrix, $A$ is not zero, and at least one of the vectors $b_{i}, i \in \mathcal{P}$, is a monomial vector.

Proof: If the positive switched system (3) is reachable, then, in particular, it is monomially reachable and hence, by Proposition 1, the (non-switched) positive system (1), with $B=\left[\begin{array}{llll}b_{1} & b_{2} & \ldots & b_{p}\end{array}\right]$, is reachable. This implies (see Lemma 2 in [16]) that $\left[\begin{array}{lllll}A & b_{1} & b_{2} & \ldots & b_{p}\end{array}\right]$ includes an $n \times n$ monomial matrix. On the other hand, if none of the vectors $b_{i}$ 's would be monomial, then $A$ should be monomial and hence it could be expressed as $A=D P$ for some nonsingular diagonal matrix $D \in \mathbb{R}_{+}^{n \times n}$ and some permutation matrix $P \in \mathbb{R}_{+}^{n \times n}$. But then, none of the vectors $A^{h} b_{j}$ could be monomial, for any $h \in \mathbb{Z}_{+}$and any $j \in \mathcal{P}$. So, none of the columns of $\mathcal{R}_{n}\left(A,\left[\begin{array}{llll}b_{1} & b_{2} & \ldots & b_{p}\end{array}\right]\right)$ would be a monomial vector, a contradiction. Finally, if $A$ would be zero, then only monomial vectors could be reached, thus contradicting the reachability assumption.

\section{REACHABILITY OF A CLASS OF DISCRETE-TIME SINGLE-INPUT POSITIVE SWITCHED SYSTEMS}

We now address the general problem of reachability for the specific class of positive switched systems (3). In the sequel, we will steadily assume, for the sake of simplicity, $\mathcal{P}=\langle 2\rangle$. The extension to the general case, when $|\mathcal{P}|=p \geq 2$, is not conceptually different, but requires a lengthy analysis of the various cases, thus making the paper quite heavy.

As a first step, we investigate the case when the system dimension is $n=2$, and later move to the case of a generic $n \in \mathbb{N}$. Reachability, as we will see, is a structural property for this specific class of systems. However, this is not, in our opinion, immediately obvious, and hence we will assume that the matrices $A, b_{1}$ and $b_{2}$ have entries in $\mathbb{R}_{+}$(and not in $\{0,1\}$, as it would be convenient if we would take care only of the nonzero patterns of the matrices and the vectors involved). Nonetheless, every time either $b_{1}$ or $b_{2}$ will be monomial, we will assume w.l.o.g. that it is a canonical vector. Also, since reachability of system (3) implies (see Lemma 1) that at least one between $b_{1}$ and $b_{2}$ is monomial, in the following we will steadily assume that $b_{1}$ is a monomial vector and, specifically, $b_{1}=\mathbf{e}_{1}$ (as we can always reduce ourselves to this case by means of a suitable permutation of the state vector entries). Also, $A$ will be positive (i.e., not zero). Finally, we will steadily assume that $b_{1}$ and $b_{2}$ are linearly independent (in particular, nonzero): were it not the case, reachability of the positive switched system (3) would reduce to the reachability of a single-input positive system (1).

Proposition 3: Consider a discrete-time single-input positive switched system described by the state equation (3), with $\mathcal{P}=\langle 2\rangle, A \in \mathbb{R}_{+}^{2 \times 2}, b_{1}=\mathbf{e}_{1}, b_{2} \in \mathbb{R}_{+}^{2}$. A necessary and sufficient condition for the system to be reachable is that

i) if $\left[\begin{array}{ll}b_{1} & b_{2}\end{array}\right]$ is a $2 \times 2$ monomial matrix, then $A$ is not the zero matrix;

ii) if only $b_{1}=\mathbf{e}_{1}$ is monomial, then $A=\left[\begin{array}{cc}0 & 0 \\ a_{21} & a_{22}\end{array}\right]$ with $\left(a_{21}, a_{22}\right) \neq(0,0)$.

Proof: Suppose, first, that $\left[\begin{array}{ll}b_{1} & b_{2}\end{array}\right]=I_{2}$. If at least one column of $A$, say the first, for instance, is strictly positive, then

$$
\begin{aligned}
& \text { Cone }\left(\left[\begin{array}{ll}
b_{1} & A b_{1}
\end{array}\right]\right) \cup \operatorname{Cone}\left(\left[\begin{array}{ll}
b_{2} & A b_{1}
\end{array}\right]\right) \\
& =\text { Cone }\left(\left[\begin{array}{ll}
1 & a_{11} \\
0 & a_{21}
\end{array}\right]\right) \cup \operatorname{Cone}\left(\left[\begin{array}{ll}
0 & a_{11} \\
1 & a_{21}
\end{array}\right]\right)=\mathbb{R}_{+}^{2} .
\end{aligned}
$$

If neither of the columns of $A$ is strictly positive, but $A \neq 0$, then there must be at least one nonzero entry in $A$. If it is on the main diagonal, then

$$
\begin{aligned}
& \text { Cone }\left(\left[\begin{array}{ll}
b_{2} & A b_{1}
\end{array}\right]\right)=\mathbb{R}_{+}^{2}, \quad \text { if } \quad a_{11}>0 \text {, } \\
& \text { Cone }\left(\left[\begin{array}{ll}
b_{1} & A b_{2}
\end{array}\right]\right)=\mathbb{R}_{+}^{2}, \quad \text { if } \quad a_{22}>0 \text {. }
\end{aligned}
$$


On the other hand, if the nonzero entry is not on the main diagonal, then either $\left(A, b_{1}\right)$ is reachable (if $a_{21}>0$ ) or $\left(A, b_{2}\right)$ is reachable (if $\left.a_{12}>0\right)$. Finally, if $A$ would be zero, then only monomial vectors would be reachable.

Suppose, now, that $b_{2}$ is not a monomial vector, then in order to reach a 2 nd monomial vector the only possibility is that $A=\left[\begin{array}{cc}0 & 0 \\ a_{21} & a_{22}\end{array}\right]$ and at least one between $a_{21}$ and $a_{22}$ is nonzero. If so,

$$
\text { Cone }\left(\left[\begin{array}{ll}
b_{1} & A b_{2}
\end{array}\right]\right)=\mathbb{R}_{+}^{2} .
$$

No other possibility arising, the proposition's statement is proved.

We now move to the analysis of reachability for the class of single-input systems (3), with $\mathcal{P}=\langle 2\rangle$ and $n>2$. The case when only $b_{1}$ is a monomial vector will be addressed in Theorem 1, while the situation when both $b_{1}$ and $b_{2}$ are monomials will be dealt with in Theorem 2 .

Theorem 1: Consider a discrete-time single-input positive switched system described by the state equation (3), with $\mathcal{P}=\langle 2\rangle, A \in \mathbb{R}^{n \times n}, b_{1}, b_{2} \in \mathbb{R}_{+}^{n}, b_{1}$ is monomial and $b_{2}$ a nonzero non-monomial vector. Suppose, also, that $\left(A, b_{1}\right)$ is not reachable ${ }^{2}$. A necessary and sufficient condition for the system to be reachable is that there exist $r \in \mathbb{N}$, and a permutation matrix $P$, such that

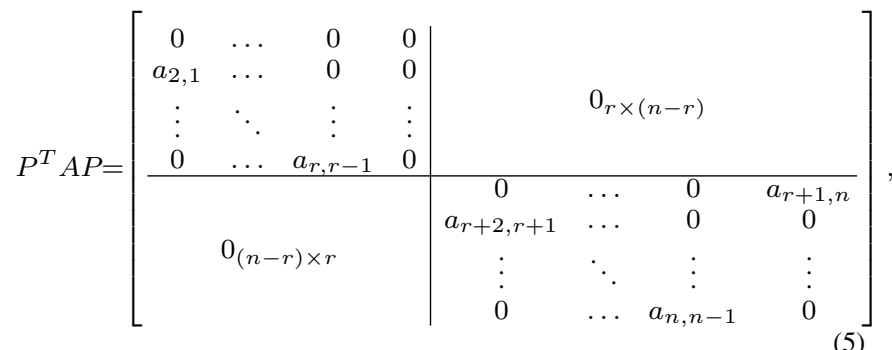

where all $a_{i+1, i}$ are positive, and (possibly after a rescaling) $P^{T} b_{1}=\mathbf{e}_{1}$, while $P^{T} b_{2}=\mathbf{e}_{r+1}+\sum_{i=1}^{r} \alpha_{i} \mathbf{e}_{i}$, and at least of the $\alpha_{i}$ 's is positive.

If so, the reachability index $I_{R}$ is $n$.

Proof: [Sufficiency] It entails no loss of generality assuming $P=I_{n}$. Under the previous assumptions, we can ensure the existence of a switching sequence $\sigma$ along which all monomial vectors can be reached. Indeed, it is easily seen that

$$
\overline{\mathrm{ZP}}\left(b_{1}\right)=\{1\}, \overline{\mathrm{ZP}}\left(A b_{1}\right)=\{2\}, \ldots \overline{\mathrm{ZP}}\left(A^{r-1} b_{1}\right)=\{r\},
$$

while the sequence of vectors

$$
A^{r} b_{2}, A^{r+1} b_{2}, \ldots, A^{r+(n-r-1)} b_{2}=A^{n-1} b_{2},
$$

consists of $n-r$ monomial vectors, each of them having a distinct nonzero pattern $\{i\}$ with $i \in\{r+1, r+2, \ldots, n\}$. As a consequence, by choosing $\sigma$ of length $n$, taking value

\footnotetext{
${ }^{2}$ Surely, by the well-know characterization of single-input positive reachable systems [8], $\left(A, b_{2}\right)$ is not.
}

2 at $k=0,1, \ldots, n-r-1$ and taking value 1 at $k=$ $n-r, n-r+1, \ldots, n-1$, we get the reachability matrix

$$
\mathcal{R}_{n}(\sigma)=\left[\begin{array}{llllll}
A^{n-1} b_{2} & \ldots & A^{r} b_{2} & A^{r-1} b_{1} & \ldots & b_{1}
\end{array}\right]
$$

which is an $n \times n$ monomial matrix, thus ensuring (by Proposition 2) reachability.

This part also proves that $I_{R}=n$.

[Necessity] Notice, preliminarily, that, by Lemma 1, $\left[\begin{array}{lll}A & b_{1} & b_{2}\end{array}\right]$ includes an $n \times n$ monomial matrix and since $b_{1}$ is monomial, but $b_{2}$ is not, $A$ must include $n-1$ linearly independent monomial vectors with nonzero pattern different from $\overline{\mathrm{ZP}}\left(b_{1}\right)$.

Consider, now, the sequence $\left\{A^{h} b_{1}, h \in \mathbb{Z}_{+}\right\}$, and set $r:=\min \left\{h \in \mathbb{N}: A^{h} b_{1}\right.$ is not a monomial vector linearly independent of $\left.b_{1}, A b_{1}, \ldots, A^{h-1} b_{1}\right\}$. By resorting to a suitable relabeling, we can always assume that $\overline{\mathrm{ZP}}\left(A^{h} b_{1}\right)=\{h+1\}, h=0,1, \ldots, r-1$, and hence we can reduce ourselves, possibly after a permutation, to the following situation:

$$
A=\left[\begin{array}{cccc|c|c}
0 & \ldots & 0 & 0 & & \\
a_{2,1} & \ldots & 0 & 0 \\
\vdots & \ddots & \vdots & \vdots & v_{1} & 0_{r \times(n-r)} \\
0 & \ldots & a_{r-1, r-2} & 0 & & \\
0 & \ldots & 0 & a_{r, r-1} & & \\
\hline 0 & \ldots & 0 & 0 & & \\
0 & \ldots & 0 & 0 & & \\
0 & \cdots & 0 & 0 & v_{2} & D \\
\vdots & & \vdots & \vdots & & \\
0 & \ldots & 0 & 0 & &
\end{array}\right]
$$

where $a_{i+1, i}>0$ for every index $i, v_{1}$ and $v_{2}$ are nonnegative vectors (notice that $A^{r} b_{1}=\left[\begin{array}{l}v_{1} \\ v_{2}\end{array}\right]$ ), $D$ is a diagonal matrix with positive entries, while $\tilde{P}$ is an $(n-r) \times(n-r)$ permutation matrix. Indeed, $A$ must include, among its columns, the $i$ th monomial vectors for $i \in\{r+1, r+2, \ldots, n\}$, and since $A^{r} b_{1}$ is not a $i$ th monomial of that type, this is the only possible solution.

It is clear that there is no way to obtain the $i$ th monomial vectors for $i>r$, by resorting to the sequence $\left\{A^{h} b_{1}, h \geq\right.$ $r\}$. In fact, since $A^{r} b_{1}$ is not a monomial vector linearly independent of $\left\{\mathbf{e}_{1}, \mathbf{e}_{2}, \ldots, \mathbf{e}_{r}\right\}$, the following cases may occur:
a) $v_{1}=0, v_{2}=0$
b) $v_{1} \neq 0$;
c) $v_{1}=0$ and $\left|\overline{\mathrm{ZP}}\left(v_{2}\right)\right|>1$.

In the first case all vectors in $\left\{A^{h} b_{1}, h \geq r\right\}$ are zero. In the second case the nonzero pattern of all vectors in $\left\{A^{h} b_{1}, h \geq\right.$ $r\}$ intersects $\langle r\rangle$. In the third case the nonzero pattern of all vectors in $\left\{A^{h} b_{1}, h \geq r\right\}$ has cardinality at least two. So, we have to find the $i$ th monomial vectors, $i>r$, in the sequence $\left\{A^{h} b_{2}, h \in \mathbb{Z}_{+}\right\}$. 
As $\left|\overline{\mathrm{ZP}}\left(b_{2}\right)\right|>1$, it is easily seen that both in case b) and in case $c)$, for every choice of $\overline{\mathrm{ZP}}\left(b_{2}\right)$, it turns out that $\left|\overline{\mathrm{ZP}}\left(A^{h} b_{2}\right)\right|>1$ for every $h \in \mathbb{Z}_{+}$. So, $\left|\overline{\mathrm{ZP}}\left(b_{2}\right)\right|>1$ is compatible only with case a). When so, it must be $\mid \overline{\mathrm{ZP}}\left(b_{2}\right) \cap$ $\{r+1, r+2, \ldots, n\} \mid=1$. If we assume w.l.o.g. that $\overline{\mathrm{ZP}}\left(b_{2}\right) \cap$ $\{r+1, r+2, \ldots, n\}=\{r+1\}$, then $\overline{\mathrm{ZP}}\left(b_{2}\right) \cap\langle r\rangle \neq \emptyset$, and the only way to generate all canonical vectors $\mathbf{e}_{i}, i>r$, is by imposing to $\tilde{P}$ a cyclic structure, thus getting the form given in (5).

Theorem 2: Consider a discrete-time single-input positive switched system described by the state equation (3), with $\mathcal{P}=\langle 2\rangle, A \in \mathbb{R}^{n \times n}, b_{1}, b_{2} \in \mathbb{R}_{+}^{n}, b_{1}$ and $b_{2}$ two linearly independent monomial vectors. Suppose that neither $\left(A, b_{1}\right)$ nor $\left(A, b_{2}\right)$ are reachable subsystems. A necessary and sufficient condition for the system to be reachable is that there exist $r \in \mathbb{N}$, and a permutation matrix $P$, such that $P^{T} A P$ takes the form

$\left[\begin{array}{cccc|cccc}0 & \ldots & 0 & a_{1 r} & 0 & \ldots & 0 & a_{1 n} \\ a_{21} & \ldots & 0 & a_{2 r} & 0 & \ldots & 0 & 0 \\ \vdots & \ddots & \vdots & \vdots & \vdots & \ddots & \vdots & \vdots \\ 0 & \ldots & a_{r, r-1} & a_{r r} & 0 & \ldots & 0 & 0 \\ \hline & & & & & & \\ \hline 0 & \ldots & 0 & a_{r+1, r} & 0 & \ldots & 0 & a_{r+1, n} \\ 0 & \ldots & 0 & a_{r+2, r} & a_{r+2, r+1} & \ldots & 0 & 0 \\ \vdots & \ddots & \vdots & \vdots & \vdots & \ddots & \vdots & \vdots \\ 0 & \ldots & 0 & a_{n r} & 0 & \ldots & a_{n, n-1} & 0 \\ & & & & & & & \end{array}\right]$

where all entries $a_{i+1, i}$ for $i=1,2, \ldots, r-1$ and $i=$ $r+1, r+2, \ldots, n-1$ are positive, at least one between $a_{1 n}$ and $a_{r+1, n}$ is positive, and (possibly after a rescaling) $P^{T} b_{1}=\mathbf{e}_{1}$ and $P^{T} b_{2}=\mathbf{e}_{r+1}$. If so, $I_{R}=n$.

Proof: [Necessity] Reachability implies monomial reachability of the switched system (3) and hence the reachability of the pair $\left(A,\left[\begin{array}{ll}b_{1} & b_{2}\end{array}\right]\right)$. So, since both $b_{1}$ and $b_{2}$ are monomial vectors (but neither of the pairs $\left(A, b_{i}\right), i=1,2$, is reachable), an index $r \in \mathbb{N}$ can be found [16] so that, after a suitable permutation, we can assume $b_{1}=\mathbf{e}_{1}, b_{2}=\mathbf{e}_{r+1}$ and $A$ takes the form

$$
\left[\begin{array}{cccc|cccc}
0 & \ldots & 0 & a_{1 r} & 0 & \ldots & 0 & a_{1 n} \\
a_{21} & \ldots & 0 & a_{2 r} & 0 & \ldots & 0 & a_{2 n} \\
\vdots & \ddots & \vdots & \vdots & \vdots & \ddots & \vdots & \vdots \\
0 & \ldots & a_{r r-1} & a_{r r} & 0 & \ldots & 0 & a_{r n} \\
\hline & & & & & & & \\
\hline 0 & \ldots & 0 & a_{r+1 r} & 0 & \ldots & 0 & a_{r+1 n} \\
0 & \ldots & 0 & a_{r+2 r} & a_{r+2 r+1} & \ldots & 0 & a_{r+2 n} \\
\vdots & \ddots & \vdots & \vdots & \vdots & \ddots & \vdots & \vdots \\
0 & \ldots & 0 & a_{n r} & 0 & \ldots & a_{n n-1} & a_{n n}
\end{array}\right]
$$

On the other hand, reachability ensures that all vectors $v>$ 0 , with $\overline{\mathrm{ZP}}(v)=\{1, r+1\}$ can be reached, and hence they belong to the cone generated by some reachability matrix

$$
\left[\begin{array}{lllll}
A^{k-1} b_{i_{k}} & A^{k-2} b_{i_{k-1}} & \ldots & A b_{i_{2}} & b_{i_{1}}
\end{array}\right],
$$

where $k \in \mathbb{N}, i_{1}, i_{2}, \ldots, i_{k} \in \mathcal{P}$. This implies that there exists $h \in \mathbb{Z}_{+}, 2 \leq h \leq k$, and $i_{h} \in \mathcal{P}$ such that $\emptyset \neq \overline{\mathrm{ZP}}\left(A^{h-1} b_{i_{h}}\right) \subseteq\{1, r+1\}$. But this necessarily implies that there exists a nonzero column in $A$ with nonzero pattern included in $\{1, r+1\}$. Since there are only two columns (the $r$ th and the $n$ th) to which we may assign this structure, then, possibly by inverting the roles of $b_{1}$ and $b_{2}$, we can always assume that it is the $n$th column.

[Sufficiency] Suppose that $b_{1}=\mathbf{e}_{1}, b_{2}=\mathbf{e}_{r+1}$ and that $A$ is described as in (6) (so, for the sake of simplicity, we assume $\left.P=I_{n}\right)$. Four cases possibly arise:

(1) $a_{1 n}=0$ (and $a_{r+1, n}>0$ );

(2) $a_{r+1, n}=0$ (and $a_{1, n}>0$ );

(3) $a_{1 n}>0, a_{r_{+}, n}>0$ and $r \leq n-r$;

(4) $a_{1 n}>0, a_{r_{+} 1, n}>0$ and $r>n-r$.

Case (1): By making use of just the same reasoning we resorted to in proving the Sufficiency part of Theorem 1, we can choose the sequence $\sigma$ of length $n$, taking value 2 at $k=0,1, \ldots, n-r-1$ and taking value 1 at $k=$ $n-r, n-r+1, \ldots, n-1$, thus getting the reachability matrix

$$
\mathcal{R}_{n}(\sigma)=\left[\begin{array}{llllll}
A^{n-1} b_{2} & \ldots & A^{r} b_{2} & A^{r-1} b_{1} & \ldots & b_{1}
\end{array}\right]
$$

which is an $n \times n$ monomial matrix. This ensures reachability.

Case (2): If so, $\left(A, b_{2}\right)$ would be reachable, thus contradicting the theorem's assumptions. So, this case cannot occur.

Case (3): Under this assumption, we preliminary notice that (we assume that all nonzero coefficients are equal to one since, as we will see, they are not relevant for the logic of the proof)

$$
\begin{aligned}
& b_{1}=\mathbf{e}_{1}, \quad b_{2}=\mathbf{e}_{r+1}, \\
& A b_{1}=\mathbf{e}_{2}, \quad A b_{2}=\mathbf{e}_{r+2}, \\
& A^{2} b_{1}=\mathbf{e}_{3}, \quad A^{2} b_{2}=\mathbf{e}_{r+3},
\end{aligned}
$$

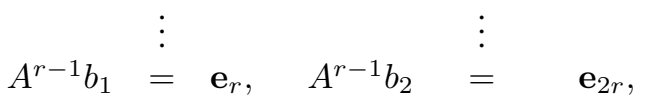

$$
\begin{aligned}
& A^{r} b_{2}=\mathbf{e}_{2 r+1}
\end{aligned}
$$

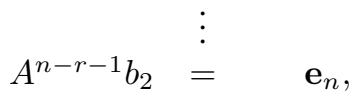

$$
\begin{aligned}
& A^{n-r} b_{2}=\mathbf{e}_{1}+\mathbf{e}_{r+1}, \\
& A^{n-r+1} b_{2}=\mathbf{e}_{2}+\mathbf{e}_{r+2}, \\
& A^{n-r+2} b_{2}=\mathbf{e}_{3}+\mathbf{e}_{r+3}, \\
& A^{n-1} b_{2}=\mathbf{e}_{r}+\mathbf{e}_{2 r}
\end{aligned}
$$

where we have exploited the fact that $r \leq n-r$.

It is not difficult to verify that every vector $v$ with $\overline{\mathrm{ZP}}(v)=\{p, p+r\}, p \in\langle r\rangle$, belongs either to $\operatorname{Cone}\left(\left[A^{p-1} b_{1} \quad A^{n-r+p-1} b_{2}\right]\right)$ or to Cone $\left(\left[\begin{array}{ll}A^{p-1} b_{2} & A^{n-r+p-1} b_{2}\end{array}\right]\right)$, depending on the specific values of its nonzero entries. Consequently, every vector 
$v \in \mathbb{R}_{+}^{n}$ belongs to the cone of a specific reachability matrix that, once reordered in the most convenient way, is thus composed:

$$
\begin{aligned}
& {\left[\begin{array}{llllllll}
b_{i_{1}} & A^{n-r} b_{2} & \mid & A b_{i_{2}} & A^{n-r+1} b_{2} & \mid & \ldots \\
\mid & A^{r-1} b_{i_{r}} & A^{n-1} b_{2} & \| & A^{r} b_{2} & \ldots & A^{n-r-1} b_{2}
\end{array}\right]}
\end{aligned}
$$

where each index $i_{p} \in \mathcal{P}$ is chosen depending on the specific subvector $\left[[v]_{p} \quad[v]_{p+r}\right]^{T}$.

Case (4): We suppose, now, that $r>s:=n-r$. In this case we face a more complicate situation as far as the vectors generated by the two sequences $\left\{A^{j-1} b_{1}, j=1,2, \ldots, r\right\}$ and $\left\{A^{j-1} b_{2}, j=1,2, \ldots, n\right\}$ are concerned. Indeed, we obtain (by neglecting again the specific values of the positive coefficients):

$$
\begin{aligned}
A^{j-1} b_{1} & =\mathbf{e}_{j}, \quad j=1,2, \ldots, r, \\
A^{t s+p-1} b_{2} & =\mathbf{e}_{p+r}+\sum_{h=0}^{t-1} \mathbf{e}_{h s+p},
\end{aligned}
$$

where we have exploited the fact that every $j \in\{1,2, \ldots, n\}$ can be expressed as $j=t s+p$, for some $t \in \mathbb{Z}_{+}$and some $p \in\{1,2, \ldots, s\}$.

Let $v^{(p)}, p \in\{1,2, \ldots, s\}$, be any positive vector with

$$
\overline{\mathrm{ZP}}\left(v^{(p)}\right) \subseteq\{p, s+p, 2 s+p, \ldots, k s+p ; r+p\},
$$

where $k$ is a suitable nonnegative number, and $k s+p \leq r$. We want to prove the following Claim:

- if $\overline{\mathrm{ZP}}\left(v^{(p)}\right) \subsetneq\{p, s+p, 2 s+p, \ldots, k s+p ; r+p\}$, then there exist indices $i_{1}, i_{2}, \ldots, i_{k+1} \in \mathcal{P}$ such that $v^{(p)}$ belongs to the cone generated by some reachability matrix of the following type

$$
\left[\begin{array}{llll}
A^{p-1} b_{i_{1}} & A^{p-1+s} b_{i_{2}} & \ldots & A^{p-1+k s} b_{i_{k+1}}
\end{array}\right]
$$

- if $\overline{\mathrm{ZP}}\left(v^{(p)}\right)=\{p, s+p, 2 s+p, \ldots, k s+p ; r+p\}$, then there exist indices $i_{1}, i_{2}, \ldots, i_{k+2} \in \mathcal{P}$ such that $v^{(p)}$ belongs to the cone generated by some reachability matrix of the following type

$$
\left[\begin{array}{llll}
A^{p-1} b_{i_{1}} & A^{p-1+s} b_{i_{2}} & \ldots & A^{p-1+(k+1) s} b_{i_{k+2}}
\end{array}\right] .
$$

To this end, we proceed by induction on the nonnegative integer $k$. Suppose, first, $k=0$. This means that $\overline{\mathrm{ZP}}\left(v^{(p)}\right) \subseteq\{p ; r+p\}$. So, if $\overline{\mathrm{ZP}}\left(v^{(p)}\right) \subsetneq\{p ; r+p\}$, then $v^{(p)}$ belongs either to Cone $\left(A^{p-1} b_{1}\right)$, if $\overline{\mathrm{ZP}}\left(v^{(p)}\right)=$ $\{p\}$, or to Cone $\left(A^{p-1} b_{2}\right)$, if $\overline{\mathrm{ZP}}\left(v^{(p)}\right)=\{p+r\}$. On the other hand, if $\overline{\mathrm{ZP}}\left(v^{(p)}\right)=\{p ; r+p\}$, then $v^{(p)}$ belongs either to Cone $\left(\left[\begin{array}{ll}A^{p-1} b_{1} & A^{p-1+s} b_{2}\end{array}\right]\right)$ or to Cone $\left(\left[\begin{array}{ll}A^{p-1} b_{2} & A^{p-1+s} b_{2}\end{array}\right]\right)$, depending on the specific values of the two nonzero entries. So, the result holds for $k=0$.

Suppose, now, that the two previous statements are verified for every vector $w^{(p)}$ with $\overline{\mathrm{ZP}}\left(w^{(p)}\right) \subseteq\{p, s+p, 2 s+$ $p, \ldots,(k-1) s+p ; r+p\}$. We want to prove that the results extend to all vectors $v^{(p)}$ with $\overline{\mathrm{ZP}}\left(v^{(p)}\right) \subseteq\{p, s+p, 2 s+$ $p, \ldots, k s+p ; r+p\}$.
To this end, suppose, first, that $\overline{\mathrm{ZP}}\left(v^{(p)}\right) \subsetneq\{p, s+p, 2 s+$ $p, \ldots, k s+p ; r+p\}$. If $k s+p \notin \overline{\mathrm{ZP}}\left(v^{(p)}\right)$, then $\overline{\mathrm{ZP}}\left(v^{(p)}\right) \subseteq$ $\{p, s+p, 2 s+p, \ldots,(k-1) s+p ; r+p\}$, and hence by the inductive assumption, $v^{(p)}$ belongs either to the cone generated by $\left[\begin{array}{llll}A^{p-1} b_{i_{1}} & A^{p-1+s} b_{i_{2}} & \ldots & A^{p-1+k s} b_{i_{k+1}}\end{array}\right]$ or to the cone generated by $\left[\begin{array}{llll}A^{p-1} b_{i_{1}} & A^{p-1+s} b_{i_{2}} & \ldots & A^{p-1+(k-1) s} b_{i_{k}}\end{array}\right]$. So, in both cases, we may claim that $v^{(p)}$ belongs to the cone generated by $\left[\begin{array}{llll}A^{p-1} b_{i_{1}} & A^{p-1+s} b_{i_{2}} & \ldots & A^{p-1+k s} b_{i_{k+1}}\end{array}\right]$ for suitable choices of the indices. On the other hand, if $k s+p \in \overline{\mathrm{ZP}}\left(v^{(p)}\right)$, then there exists $\alpha>0$ such that

$$
w^{(p)}=v^{(p)}-\alpha A^{p-1+k s} b_{1},
$$

satisfies $\overline{\mathrm{ZP}}\left(w^{(p)}\right) \subsetneq\{p, s+p, 2 s+p, \ldots,(k-1) s+p ; r+p\}$, and hence by resorting to the inductive assumption, again, we may say that $v^{(p)}$ belongs to the cone generated by

$$
\left[\begin{array}{lllll}
A^{p-1} b_{i_{1}} & A^{p-1+s} b_{i_{2}} & \ldots & A^{p-1+(k-1) s} b_{i_{k}} & A^{p-1+k s} b_{1}
\end{array}\right] .
$$

Assume, now, that $\overline{\mathrm{ZP}}\left(v^{(p)}\right)=\{p, s+p, 2 s+p, \ldots, k s+$ $p ; r+p\}$. Then there exists $\alpha>0$ such that

$$
w^{(p)}=v^{(p)}-\alpha A^{p-1+(k+1) s} b_{2}
$$

satisfies $\overline{\mathrm{ZP}}\left(w^{(p)}\right) \subsetneq\{p, s+p, 2 s+p, \ldots, k s+p ; r+p\}$. So, by the previous part of the proof, we can claim that $w^{(p)}$ belongs to the cone generated by some matrix

$$
\left[\begin{array}{llll}
A^{p-1} b_{i_{1}} & A^{p-1+s} b_{i_{2}} & \ldots & A^{p-1+k s} b_{i_{k+1}}
\end{array}\right],
$$

and this implies that $v^{(p)}$ belongs to some cone

$$
\left[\begin{array}{lllll}
A^{p-1} b_{i_{1}} & A^{p-1+s} b_{i_{2}} & \ldots & A^{p-1+k s} b_{i_{k+1}} & A^{p-1+(k+1) s} b_{2}
\end{array}\right],
$$

thus completing the proof by induction of our Claim.

To conclude, now that we have shown that every vector $v^{(p)}, p \in\{1,2, \ldots, s\}$, with $\overline{\mathrm{ZP}}\left(v^{(p)}\right) \subseteq\{p, s+p, 2 s+$ $p, \ldots, k s+p ; r+p\}$, where $k$ is a suitable nonnegative number, belongs to the cone generated by some reachability matrix of the following type

$$
\left[\begin{array}{llll}
A^{p-1} b_{i_{1}} & A^{p-1+s} b_{i_{2}} & \ldots & A^{p-1+(k+1) s} b_{i_{k+2}}
\end{array}\right],
$$

(where $p-1+(k+1) s \leq n-1$ ), it is sufficient to notice that every positive vector $v \in \mathbb{R}_{+}^{n}$ can be expressed as $v=\sum_{p=1}^{s} v^{(p)}$, for suitable choices of the vectors $v^{(p)}$, and since each of them is obtained by combining columns where different powers of the matrix $A$ appear, it is immediately seen that we can always find indices $i_{1}, i_{2}, \ldots, i_{n} \in \mathcal{P}$ such that

$$
v \in \text { Cone }\left(\left[\begin{array}{lllll}
A^{n-1} b_{i_{n}} & \ldots & A^{2} b_{i_{3}} & A b_{i_{2}} & b_{i_{1}}
\end{array}\right]\right) .
$$

Clearly, in all four cases it turns out that $I_{R}=n$.

\section{Conclusions}

In this paper we have investigated monomial reachability and reachability for the special class of single-input positive switched systems which commute among subsystems sharing the same system matrix and different input-to-state vectors $b_{i} \in \mathbb{R}_{+}^{n}$. Monomial reachability can be reduced to the reachability of a standard positive system, and it turns out 
that the monomial reachability index $I_{M R}$ is always upper bounded by the system dimension $n$.

Reachability property has been investigated in detail for the special case when the number $p$ of subsystems among which the system commutes is 2 . In this setting, two distinct canonical forms, depending on whether both $b_{1}$ and $b_{2}$ are monomial, or only one of them is, have been derived. In both cases, it turns out that the reachability index $I_{R}$ is always equal to $n$.

\section{REFERENCES}

[1] G.P. Barker. Theory of cones. Lin. Alg. Appl., 39:263-291, 1981.

[2] A. Berman and R.J. Plemmons. Nonnegative matrices in the mathematical sciences. Academic Press, New York (NY), 1979.

[3] R. Bru, S. Romero, and E. Sanchéz. Canonical forms for positive discrete-time linear control systems. Linear Algebra \& its Appl., 310:49-71, 2000.

[4] R.A. Brualdi and H.J. Ryser. Combinatorial matrix theory. Cambridge Univ.Press, Cambridge (GB), 1991.

[5] P.G. Coxson and L.C. Larson. Monomial patterns in the sequence $A^{k}$ b. Lin. Alg. Appl., 94:89-101, 1987.

[6] P.G. Coxson and H. Shapiro. Positive reachability and controllability of positive systems. Lin. Alg. Appl., 94:35-53, 1987.

[7] M. Egerstedt and M. Babaali. On observability and reachability in a class of discrete-time switched linear systems. In Proc. of the 2005 American Control Conf., pages 1179-1180, Portland, Orgeon, 2005.

[8] L. Farina and S. Rinaldi. Positive linear systems: theory and applications. Wiley-Interscience, Series on Pure and Applied Mathematics, New York, 2000.

[9] S.S. Ge, Z. Sun, and T.H. Lee. Reachability and controllability of switched linear discrete-time systems. IEEE Trans. Aut. Contr., 46, no. 9:1437-1441, 2001.

[10] L.T. Conner Jr. and D.P. Stanford. State deadbeat response and observability in multi-modal systems. SIAM J. Contr. Optimiz., 22(4):630-644, 1984.

[11] L.T. Conner Jr. and D.P. Stanford. The structure of the controllable set for multi-modal systems. Linear Algebra \& its Appl., 95:171-180, 1987.

[12] D. Liberzon and A.S. Morse. Basic problems in stability and design of switched systems. IEEE Contr. Syst. Magazine, 19:59-70, 1999.

[13] P. Santesso and M.E. Valcher. Monomial reachability and zerocontrollability of discrete-time positive switched systems. Systems and Control Letters, 57:340-347, 2008.

[14] D.P. Stanford and L.T. Conner Jr. Controllability and stabilizability in multi-pair systems. SIAM J. Contr. Optimiz., 18(5):488-497, 1980.

[15] Z. Sun and D. Zheng. On reachability and stabilization of switched linear systems. IEEE Trans. Aut. Contr., 46, no. 2:291-295, 2001.

[16] M.E. Valcher. Controllability and reachability criteria for discrete time positive systems. Int. J. of Control, 65:511-536, 1996.

[17] Y. Wang, G. Xie, and L. Wang. Reachability of switched discrete-time systems under constrained switching. In Proc. of the 42nd IEEE Conf. on Decision and Control, pages 5765-5770, Maui, Hawaii, 2003.

[18] M.A. Wicks, P. Peleties, and R.A. De Carlo. Switched controller synthesis for the quadratic stabilization of a pair of unstable linear systems. European J. of Control, 4, no. 2:140-147, 1998.

[19] G. Xie and L. Wang. Reachability realization and stabilizability of switched linear discrete-time systems. J. Math. Anal. Appl., 280:209220, 2003 . 